\title{
Caracterización del Material de Interfaz Térmica (TIM) de un microprocesador
}

\author{
Characterization of the Thermal Interface \\ Material (TIM) of a microprocessor
}

José Alberto Salazar-Jiménez'

Fecha de recepción: 6 de febrero del 2013

Fecha de aprobación: 21 de junio del 2013

Salazar, J. Caracterización del Material de Interfaz Térmica (TIM) de un microprocesador. Tecnología en Marcha. Vol. 26, Nº 4. Pág 36-4l

Estudiante de Ingeniería en Materiales, Escuela de Ciencia e Ingeniería de los Materiales, Instituto Tecnológico de Costa Rica. Costa Rica. Correo electrónico: jasj.1991@gmail.com. Tel. 86617416. 


\section{Acerca de este trabajo}

Este trabajo corresponde al primer examen parcial del curso de Microscopía Electrónica (CM-4 I09) de la Escuela de Ciencia e Ingeniería de los Materiales del Instituto Tecnológico de Costa Rica.

\section{Palabras clave}

Material de Interfaz Térmica; microprocesador; SEM; EDX

\section{Resumen}

Los Materiales de Interfaz Térmica (TIM, por sus siglas en inglés) son aquellos que se utilizan en microelectrónica, principalmente en microprocesadores, para aumentar el rendimiento de la transferencia de calor entre el chip de silicio y el disipador de calor de cobre, reemplazando al aire presente en esta zona debido a la rugosidad de la superficie de ambos componentes. La transferencia de calor en los TIM depende principalmente de su composición y espesor. En este trabajo se determinó el espesor y la composición química del material de interfaz térmica de un microprocesador Intel Pentium 4, mediante el uso de un Microscopio de Barrido Electrónico (SEM, por sus siglas en inglés) equipado con un espectrómetro de espectroscopía de dispersión de energía de rayos $\times$ (EDX, en inglés) en el Laboratorio de Nanotecnología del Instituto Tecnológico de Costa Rica.

\section{Key words}

Thermal Interface Material; Microprocessor; SEM; EDX

\section{Abstract}

Thermal Interface Materials (TIM) are materials which are used in microelectronics, mainly in microprocessors, to improve the yield of heat transfer between the silicon die and the copper heat sink, replacing the trapped air in this area due to the surface roughness of both components. The heat transfer in this material depends mostly of its chemical composition and its thickness. This work deals with the measure of thickness and the determination of chemical composition for the TIM of an Intel Pentium 4 microprocessor, using a scanning electron microscope (SEM) equipped with energydispersive $x$-ray spectrometer (EDX), present in the Nanotechnology Laboratory of the ITCR. 


\section{Introducción}

Los dispositivos microelectrónicos, sobre todo los microprocesadores, generan cada vez más calor durante su funcionamiento, como consecuencia de la miniaturización de sus componentes y el aumento de la velocidad de procesamiento de datos (Deppisch et al., 2006). Debido a esto, un aspecto importante en el diseño de los dispositivos microelectrónicos es la extracción efectiva de dicho calor, ya que este disminuye su rendimiento y puede dañarlos.

La transferencia térmica en la zona entre el chip de silicio y el disipador de calor de cobre del microprocesador ocurre de forma ineficiente, ya que a nivel microscópico la superficie de estos posee una alta rugosidad, permitiendo la presencia de aire que queda atrapado. Para reducir la resistencia térmica en esta zona se utilizan Materiales de Interfaz Térmica (TIM, por sus siglas en inglés) para que desplacen dicho aire, ya que estos materiales tienen coeficientes de transferencia de calor mayores, mejorando en gran medida la extracción de calor en el microprocesador (Jaiswai \& Dwivedi, 20 I I). En la figura I se muestra un esquema de la ubicación del TIM en un microprocesador.

Como consecuencia a la rugosidad de los componentes donde se introduce el TIM, el espesor de este no es uniforme, de modo que se le debe determinar un espesor promedio (Bond-Line Thickness, $B L T$, por sus siglas en inglés), como se ilustra en la figura 2. En la misma figura se observa que el TIM no rellena completamente las cavidades rugosas de los componentes, por lo que siempre se presenta una pequeña cantidad de aire atrapado que no permite que la eficiencia de la transferencia de calor alcance el $100 \%$, lo que se considera uno de los problemas más importantes en el uso de TIM.
La resistencia térmica total efectiva en esta zona $R_{\text {eff }}$ corresponde a la suma de la resistencia térmica debido a la conducción en el TIM y las resistencias de contacto entre el TIM y las dos superficies de contacto, como se expresa en la siguiente ecuación (Sarvar et al., 2006):

$$
\mathrm{Re}_{\mathrm{e} f}=\frac{B L T}{k_{T I M} \cdot A}+R_{\text {cont } 1}+R_{\text {cont } 2}\left[\frac{W}{m \cdot K}\right]
$$

Donde: BLT es el espesor promedio del TIM, $k_{T M}$ es la conductividad térmica del TIM, ${ }^{A}$ es el área normal a la dirección de transferencia de calor y $R_{\text {contl }}$ y $R_{\text {contz }}$ son las resistencias térmicas de contacto. En esta ecuación se aprecia la directa dependencia de la resistencia térmica del TIM con respecto a su espesor.

Existen varios tipos de materiales de interfaz térmica, los cuales se revisan en Jaiswai \& Dwivedi (20 I I), Sarvar et al., (2006) y Chung (200 I), siendo los más utilizados a nivel comercial las pastas térmicas y los solder-TIM. Las pastas térmicas consisten en compuestos de partículas conductoras, usualmente metales $u$ óxidos de estos, dispersadas en una matriz polimérica. Mientras que los solder-TIM consisten en un metal, el cual se funde y se deposita en el microprocesador, permitiendo una alta tasa de transferencia de calor (Sarvar et al., 2006); siendo el indio el más utilizado, ya que posee relativamente un bajo punto de fusión $\left(157^{\circ} \mathrm{C}\right)$, un alto coeficiente de conductividad térmica $\left(80 \frac{\mathrm{W}}{\mathrm{m}} \cdot K\right)$ y se adapta al estrés mecánico inducido por la diferencia en expansión térmica entre el chip de silicio y el disipador de cobre (Subramanian et al., 2005).

Este trabajo tiene como objetivo determinar el espesor promedio y la composición química del TIM en un microprocesador INTEL Pentium 4.

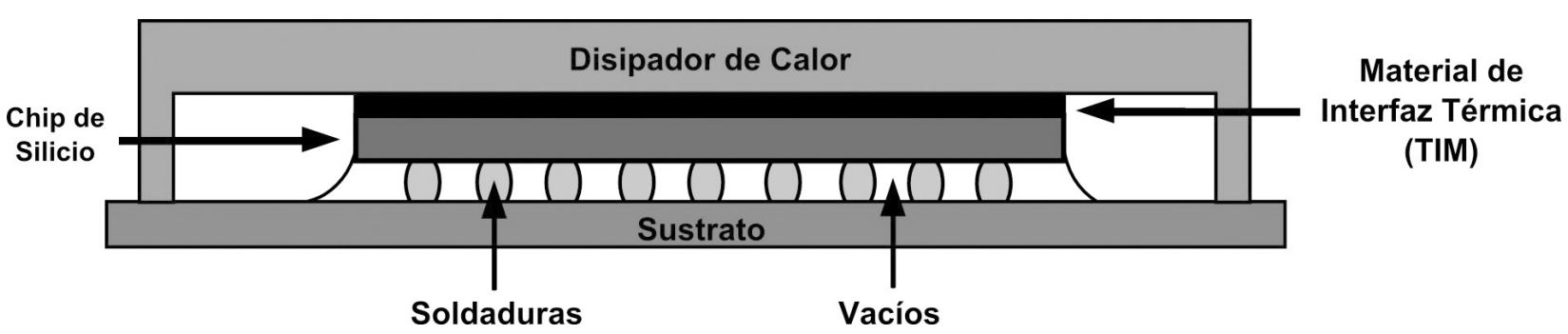

Figura I. Esquema de un microprocesador 


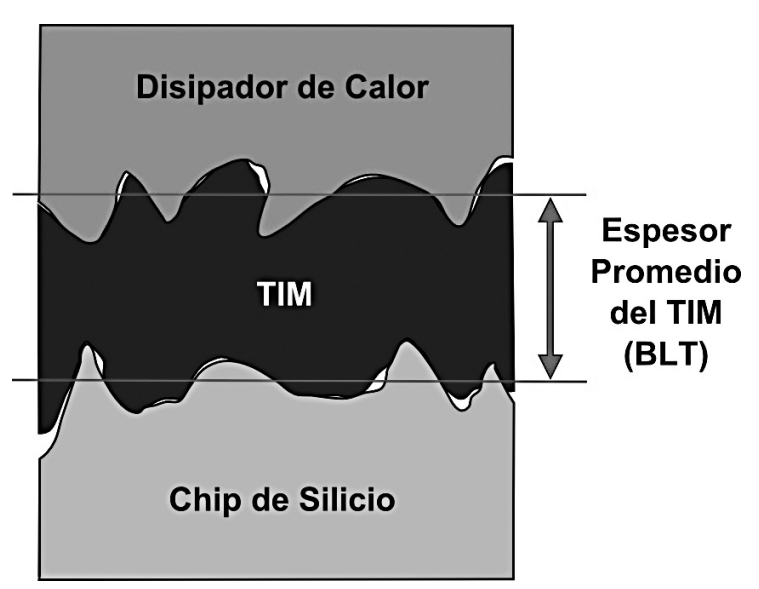

Figura 2. Espesor promedio del TIM

\section{Procedimiento experimental}

\section{Preparación de la muestra}

El microprocesador Intel Pentium 4 que se utilizó fue facilitado por la Escuela de Ingeniería en Electrónica del Instituto Tecnológico de Costa Rica (ITCR). Este fue sometido al siguiente proceso de preparación para la obtención de una muestra, en la Escuela de Ciencia e Ingeniería de los Materiales del ITCR:

I. Corte del microprocesador en dirección diagonal, a través de una cortadora Leco MSX250A. La figura 3 muestra el microprocesador con la dirección de corte señalizada; tomando cualquiera de las dos partes como muestra.

2. Pulido de la muestra con lijas de carburo de silicio número 600, 1000 y 1500, en dicho orden, para reducir las líneas producidas.

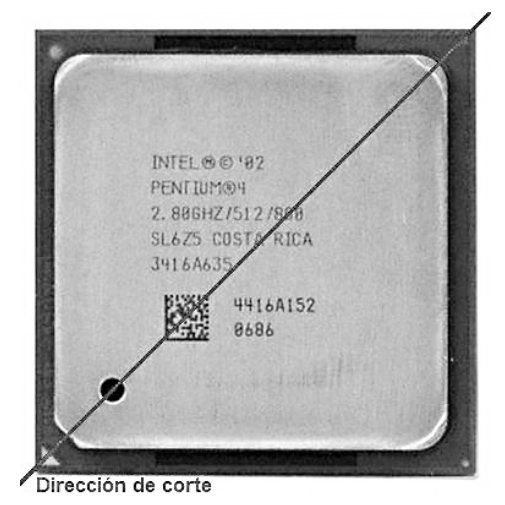

Figura 3. Dirección de corte en el microprocesador utilizado
3. Pulido de la muestra en el paño para pulido con alúmina, hasta que la superficie tenga un acabado tipo espejo, lo cual indica que el efecto de las líneas de pulido no será significativo.

\section{Caracterización de la muestra}

La apariencia de la muestra se observará mediante un microscopio óptico para comprobar su correcta preparación, al inferir que las líneas de pulido no serán significativas para el proceso de caracterización posterior. Las medidas del espesor del TIM se efectuarán a través de un Microscopio de Barrido Electrónico (SEM, por sus siglas en inglés) modelo Hitachi TMI000, mientras que la composición se determinará mediante un Espectrómetro de Dispersión de Energía de Rayos X (EDX), el cual se encuentra equipado al SEM, ambos pertenecientes al Laboratorio de Nanotecnología del ITCR.

\section{Resultados y discusión}

La figura 4 muestra una imagen obtenida con el microscopio óptico al final del proceso de preparación con un aumento de 50x, donde a esta magnitud no se observan líneas de pulido, habiendo sido reducidas en gran medida en la etapa final de preparación de la muestra de pulido en el paño; por lo cual estas no tendrán un efecto significativo a la hora de caracterizar el espesor y la composición química del material de interfaz térmica de la muestra de microprocesador. En esta figura se puede observar y delimitar con facilidad el TIM en la interface entre el chip de silicio y el disipador de calor de cobre.

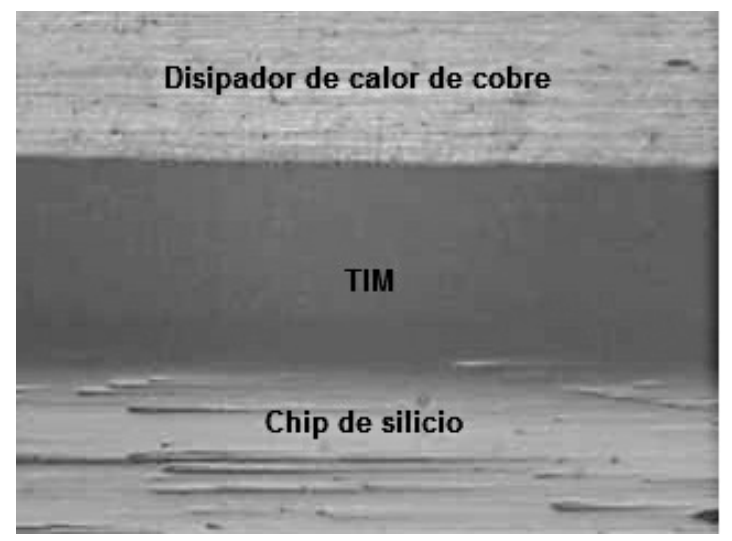

Figura 4. Imagen del TIM a 50x tomada en un microscopio óptico 
En la figura 5 se presentan tres imágenes tomadas con el microscopio de barrido electrónico con una magnificación de 150x, en las cuales se aprecian las medidas realizadas en los extremos y cerca del centro del material de interfaz térmica para determinar

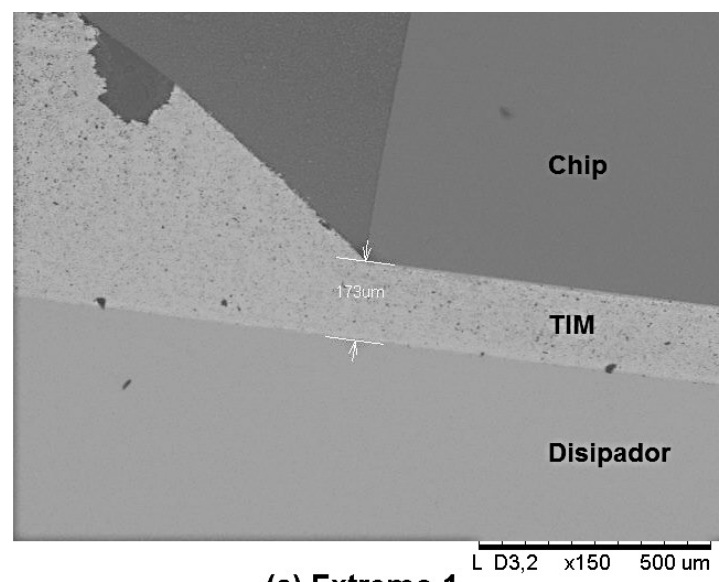

(a) Extremo 1

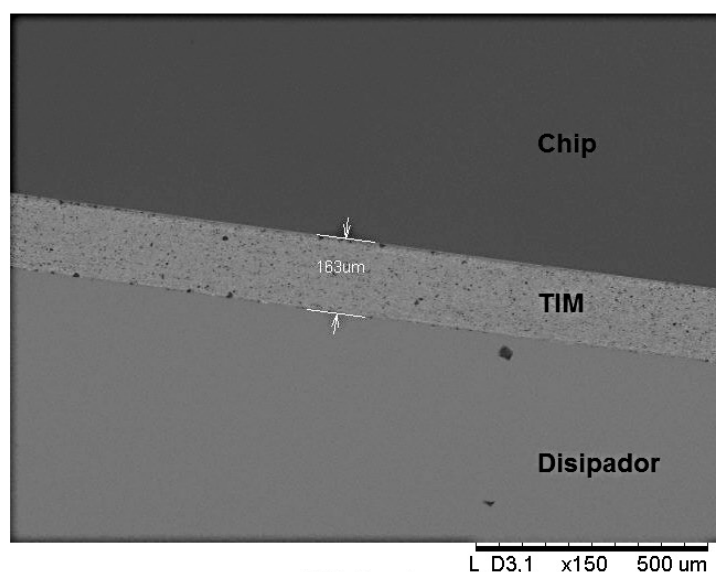

(b) Centro

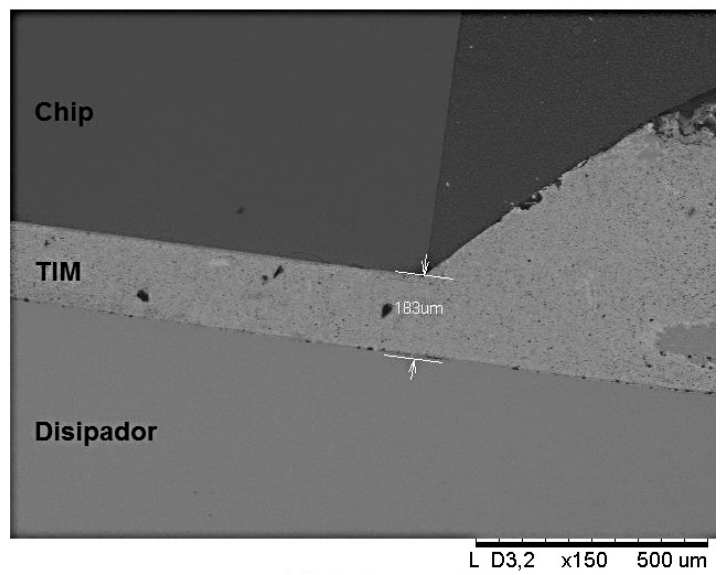

(c) Extremo 2

Figura 5. Imágenes SEM a I50x mostrando las medidas de espesor del TIM el espesor promedio de este a través de estas tres medidas, cuyo valor determinado fue de $173 \mu \mathrm{m}$. Las diferencias en los valores de las medidas del espesor del TIM se pueden haber producido principalmente por una diferencia en la distribución de la temperatura y la presión a la hora de fundirlo y colocarlo en el microprocesador durante su proceso de fabricación, provocando que el TIM no fluyera en estado fundido de forma homogénea.

En la figura 6 se muestra el espectrograma de dispersión de rayos $x$ obtenido al analizar la muestra dentro del SEM, donde el eje absciso (horizontal) representa valores de energía característicos de cada elemento químico, mientras que el eje ordenado (vertical) representa el conteo normalizado de la detección de estos valores energéticos. En este gráfico se aprecia la detección de altas cantidades de cobre y silicio, además de indio. Esto ocurrió como consecuencia de que el área de análisis que cubre el espectrómetro a la hora de utilizarlo supera la medida del espesor delTIM mismo, abarcando parte del chip de silicio y del disipador de cobre. En la figura 7 se presenta el mismo gráfico de EDX pero en términos porcentuales, donde el alto porcentaje de indio detectado (aproximadamente $11,2 \%$ ) indica que es el componente principal del material de interfaz térmica para este microprocesador, lo cual sugiere que es del tipo solder-TIM. Existen muchas empresas que manufacturan este tipo de TIM, siendo Indium Corporation (http://www.indium.com/) una de las más conocidas.

\section{Conclusiones}

El material de interfaz térmica presenta un espesor bastante regular, con un valor promedio de $173 \mu \mathrm{m}$; donde la variación de este pudo haber ocurrido por una diferencia en la distribución de la temperatura y la presión en el momento de fundirlo y colocarlo en el microprocesador durante el proceso de su manufactura.

El alto porcentaje de indio detectado mediante la técnica EDX sugiere que el material de interfaz térmica es del tipo solder-TIM a base de indio, el cual presenta muy buenas propiedades relativas a otros metales, como su bajo punto de fusión $\left(157^{\circ} \mathrm{C}\right)$ y su alta conductividad térmica $\left(80 \frac{W}{m} \cdot K\right)$. 


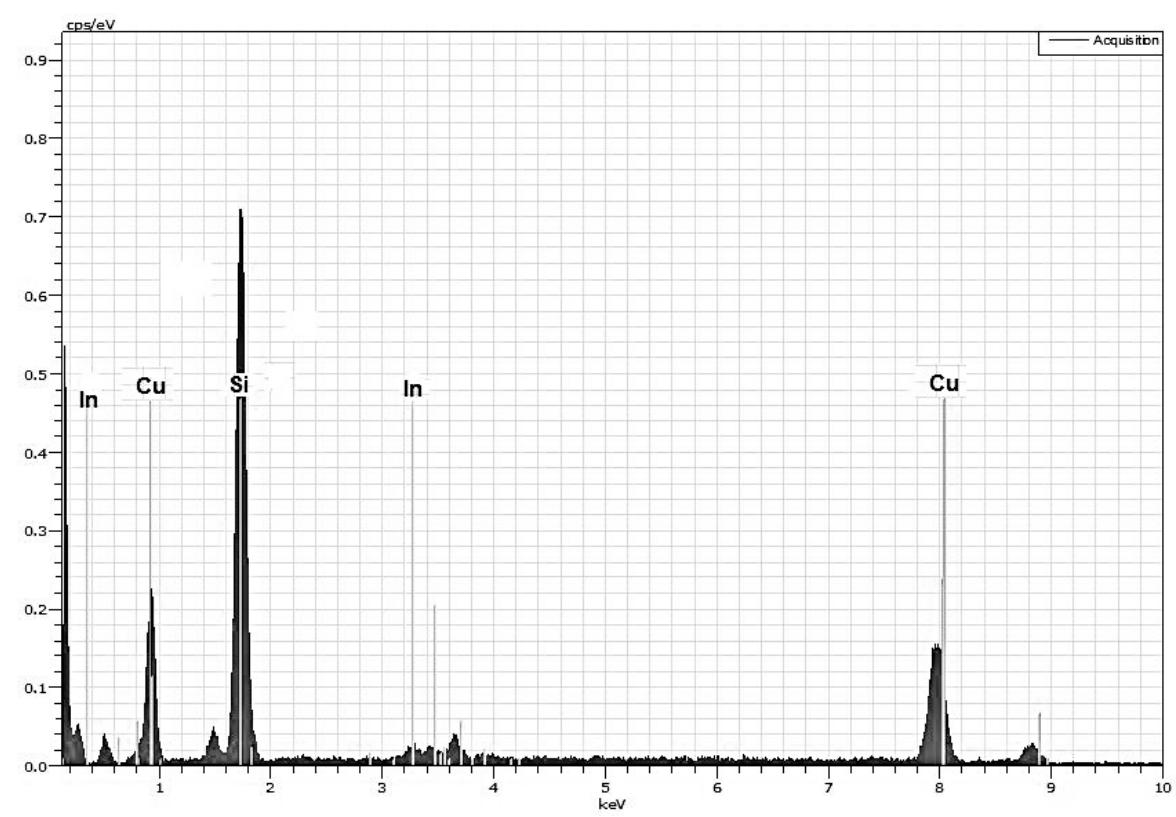

Figura 6. Espectrograma de EDX obtenido

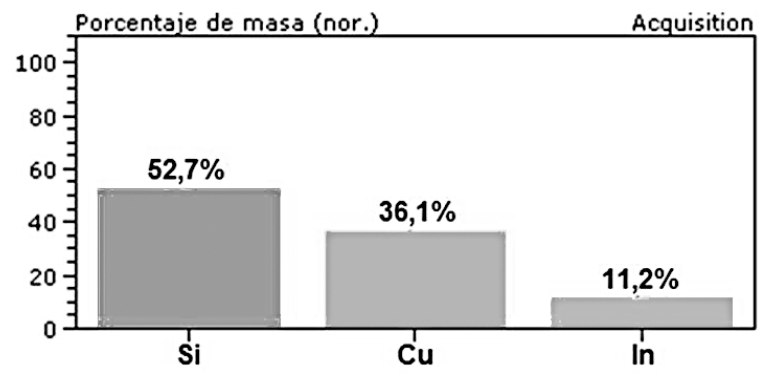

\section{Bibliografía}

Deppisch, C., Fitzgerald, T., Raman, A., Hua, F., Zhang, C., Liu, P. \& Miller, M. (2006). The Material Optimization and Reliability Characterization of an Indium-Soldier Thermal Interface Material for CPU Packaging.JOM, Vol. 58(6): 67-74. Obtenido desde http://link.springer.com/article/ |0.1007\%2Fs I | 837006-0 | 86-6? LI=true

Jaiswai, P. \& Dwivedi, C. (20II). Thermal Interface Materials used for improving the Efficiency and Power Handling Capability of Electronic Devices: A Review. International Journal of Innovative Technology and Creative Engineering, Vol.

Figura 7. Concentración de los elementos químicos detectados mediante EDX

\section{Agradecimientos}

Agradezco a la Escuela de Ciencia e Ingeniería de los Materiales del ITCR por la ayuda facilitada durante la preparación de la muestra y al Laboratorio de Nanotecnología de la misma institución por el préstamo del equipo para la caracterización de esta. I (5): I-9. Obtenido desde http://ijitce.co.uk/download/May/ IJTCE_May3.pdf

Sarvar, F., Whalley, D.C. \& Conway, P.P. (2006). Thermal Interface Materials $-A$ review of the state of art. Proceedings of the $\left.\right|^{\text {st }}$ IEEE Electronic System-Integration Technology Conference, Vol. 2, 1292-1302. Obtenido desde https://dspace.lboro. ac.uk/dspace-jspui/bitstream/2 134/4 197///RC74.pdf

Chung, D.D.L. (200I). Thermal Interface Materials. Journal of Materials Engineering and Performance, Vol. IO(I): 56-59. Obtenido desde http://link.springer.com/article/l0.1361\%2 FI0599490 | 770345358? LI=true

Subramanian, J.S., Rodgers, P., Newson, J., Rude, T., He, Z., Besnoin, E., Weihs, T.P., Eveloy, V. \& Pecht, M. (2005). Room Temperature Soldering of Microelectronic Component for Enhanced Thermal Performance. Proceedings of the $6^{\text {th }}$ IEEE International Conference on Thermal, Mechanical and Multiphysics Simulation and Experiment in Microelectronics and Microsystems, 68I-686. Obtenido desde http://www.calce. umd.edu/articles/openart/Page_2_Room_Temperature.pdf 